\title{
Design and Experiment Study of Transformer Fault Monitoring System Based on acoustic characteristics
}

\author{
Deng Kai \\ School of Electrical and Electronic Engineering, Huazhong University of Science and Technology, \\ Wuhan, Hubei 430074, China
}

Keywords: Transformer, fault monitoring, acoustic signal, system design.

\begin{abstract}
Transformers in operation is accompanied by certain acoustic signals, and the acoustic signals can reflect the running state of the transformer to a great extent. In view of the characteristics of transformer fault and the principle of acoustic signals generation, a transformer fault monitoring device based on acoustic characteristics is developed. The results of laboratory test and substation field test shows that the device can monitor and analysis the acoustic signals of transformers in operation in real time, evaluate the health of transformers, and has certain value of the field application.
\end{abstract}

\section{Introduction}

The safe and stable operation of power transformers is an important factor to ensure the reliability of power supply system, so learning the running state of transformers in time is very important to improve the reliability of transformers. On-line monitoring of power transformer condition is one of the most effective means to prevent the occurrence of accidents. A lot of research has been done on the on-line monitoring and fault diagnosis of power transformer running state.

In the operation of power transformers, the mechanical deformation is produced under the influence of the internal electric field and magnetic field. The mechanical deformation is conducted by self-structure and performs as vibration signal. The vibration signal is transmitted by the surrounding air medium, and then it produces acoustical signals. These signals can reflect the operation of the transformer to a great extent[1].

In the field inspection operation, experienced technical staff can always determine the transformer's running state and find fault type or even locate it roughly by putting the ear close to the transformer box and carefully listening to the sounds of the transformer. This widespread-used diagnosis method depends heavily on the subjective judgment and personal experience of the technical personnel, and it has a great uncertainty. But this phenomenon has proved the feasibility of the diagnosis of transformer fault by acoustic signal, and with the aid of the high sensitivity sensor and the modern digital signal processing technology, it is necessary to realize the on-line monitoring and fault diagnosis which is more objective and reliable than the human ear[2, 3].

Acoustic detection method is a dynamic and nondestructive testing method, and transformers can be on-line and real-time detected in the case of no electromagnetic interference. Based on the above advantages, this paper develops a transformer fault detection device based on acoustic signal.

\section{Theory of acoustic detection of transformers}

Under normal circumstances, transformers operate accompany by sound, and the sound is derived from the vibration of the internal components of the transformers through the medium of radiation. The vibration of the transformer on the whole involves body structure vibration, vibration of cooling device and vibration produced by switching loaded regulating tap changer. Among them, the vibration of the transformer body and the cooling device is steady, and the vibration of the switching is transient[4].

Acoustic signals contains the state information of the transformer operation. When certain parts of the unit or its operating conditions and parameters change, or internally it generates discharge sound, 
the sound of the transformer will have a certain degree of change. By processing and quantitative analysing the acoustic signal, the running state of the transformer can be detected and the faults of the transformer can be diagnosed.

\section{Design of monitoring device}

\subsection{Structure of Monitoring Device}

Based on the relevant research and analysis, the transformer fault monitoring system based on acoustic characteristics is developed. Its overall structure is shown in Figure 1. The whole system can be divided into five parts: sensor, data acquisition and processing, data communication, control center, state display and alarm.

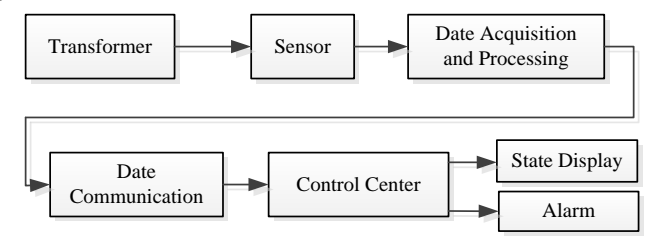

Fig. 1. The structure diagram of monitoring system

When the transformer operates normally or abnormally, the acoustic signal is collected by the sensor and processed by the terminal, and then uploaded to the control terminal in a wireless communication way. The control terminal will analyze and compare it further, and display the transformer running state information in time. If the transformer operates abnormally, it will automatically alarm and alert personnel on duty to take the relevant measures timely.

\subsection{Data Acquisition and Preprocessing Unit}

Data acquisition and preprocessing unit is the core part of the acquisition terminal, and the schematic diagram is shown in Figure 2. After the acoustic signal is collected by the sensor, the signal is converted to electrical signal and amplified by the preamplifier. Then the filter module is used to extract the signal component of each band and eliminate some low frequency or high frequency periodic interference. In addition, the signals through the device filter module can be divided into three forms: frequency signal, low frequency signal and wide band signal. Measurement can choose the form according to their needs, which reduces the signal processing burden. After the signal is filtered through the signal conditioning circuit and anti-aliasing filter, it meets the requirements of the $\mathrm{A} / \mathrm{D}$ sampling. The date is upload to the control terminal PC in wireless communication way after the completion of the scheduled A/D sampling.

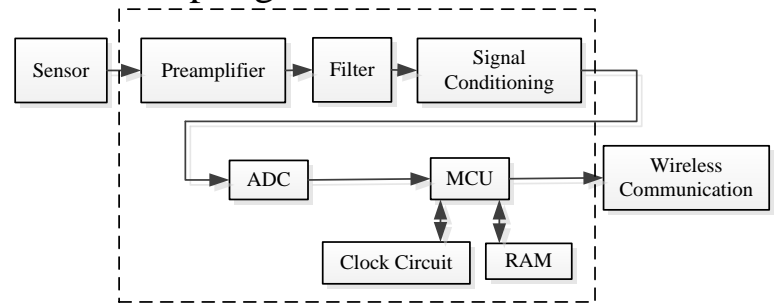

Fig. 2 The structure diagram of data acquisition and signal preprocessing unit

\subsection{Sensor Selection}

The ultrasonic signal decays easily in the transmission process, so this device mainly collect audible sound. Based on this, the device selects the piezoelectric vibration acceleration sensor. The low frequency response of this sensor is good, and it is not required to propagate through the air medium because of its contact-type measurement. Therefore, the influence of the background noise is small, the signal to noise ratio is high, the power can be provided, the installation is convenient, the service life is longer, and the use of the sensor is in accordance with the monitoring system[5].

Amplitude frequency and phase frequency characteristics of piezoelectric accelerometer are:

$$
\left|\frac{x_{m}-x}{\ddot{x}}\right|=\frac{\left(1 / \omega_{n}\right)^{2}}{\sqrt{\left[1-\left(\omega / \omega_{n}\right)^{2}\right]^{2}+4 \zeta^{2}\left(\omega / \omega_{n}\right)^{2}}}
$$




$$
\varphi=-\arg \tan \frac{2 \zeta\left(\omega / \omega_{n}\right)^{2}}{1-\left(\omega / \omega_{n}\right)^{2}}
$$

where $x_{m}$ is mass; $x$ is vibration displacement; $\omega$ is vibration angular frequency; $\omega_{n}$ is the natural frequency of the transducer.

Combined with the actual application needs and through the market survey, we finally choose ULT0188 type vibration acceleration sensor as the measurement sensor of transformer fault detection system. There is a micro IC amplifier inside of the sensor, which combines the traditional piezoelectric acceleration sensor and the charge amplifier. It can be directly connected with the recording, display and acquisition instrument, simplifies the testing system and improves the test accuracy and reliability. The sensitivity is $100 \mathrm{mv} / \mathrm{g}$, the resonant frequency is $23 \mathrm{kHz}$, the resolution is $0.002 \mathrm{~g}$, and the working temperature is $-50 \sim 121{ }^{\circ} \mathrm{C}$.

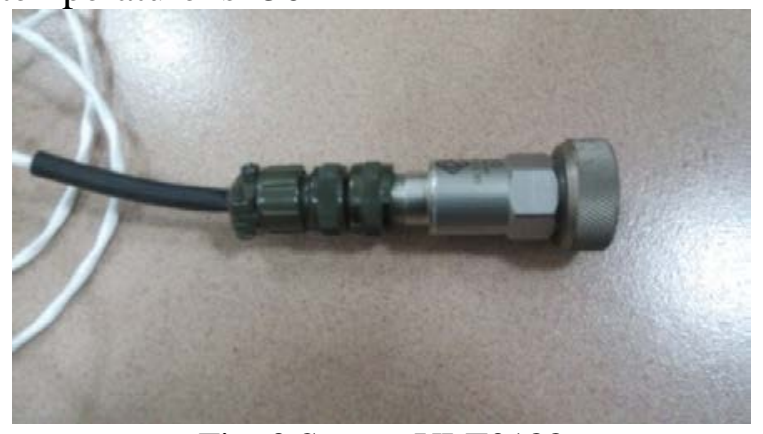

Fig. 3 Sensor-ULT0188

\subsection{AD Sampling Module}

The system selects the STM32F205RET6 chip of ST company as the control core of AD sampling. STM32 series processor is based on the Cortex-M3 ARM kernel 32 bit enhanced flash microcontroller. It has the characteristics of high integration, low power consumption, excellent performance, and it provides the functions of reset circuit, RC oscillation, low voltage detection, etc., so it can meet the needs of the signal sampling in this paper.

\subsection{Monitoring System Control Terminal}

Control terminal PC is the upper monitor of the transformer fault monitoring system, and it is responsible for the acquisition of data acquisition terminal commands and controlling data communication and signal analysis and processing. Based on the development environment GUI of MATLAB, the system has developed the control system of human-computer interaction. It can realize the serial port settings (serial number of the equipment and communication baud rate), sampling set(channel selection, sampling frequency and sampling points), operating instructions (a query from the machine, status query, communications test, sampling order) and graphic data processing function.

\section{Laboratory test}

In order to test the performance of the transformer acoustic fault monitoring device which is developed in the above test, some test is carried out in the laboratory. At present, a large proportion of discharge fault occupy a large proportion of transformer fault, so a typical discharge model inside transformer oil is designed and make, and a test platform is set up. The test platform is shown in figure 4 .

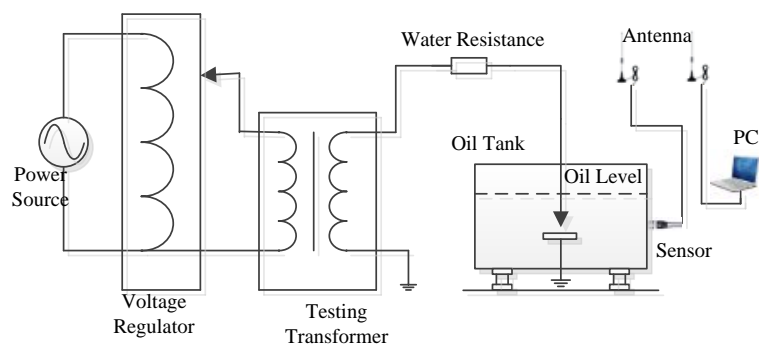

Fig. 4 Schematic of test platform of transformer oil discharge 
A needle-plate discharge model made of brass is selected so that it can simulate the discharge between the interior edges of certain components and other metal surface. The waveform of acoustic signals collected when the needle-plate gap is not breakdown and breakdown is shown in Figure 5 and Figure 6.

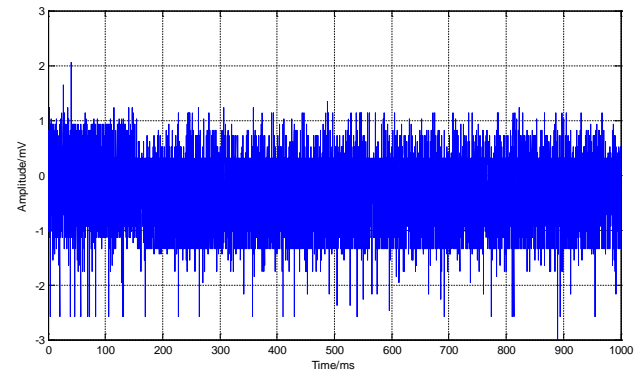

Fig. 5 Acoustic waveform when there's no discharge between needle-plate gap

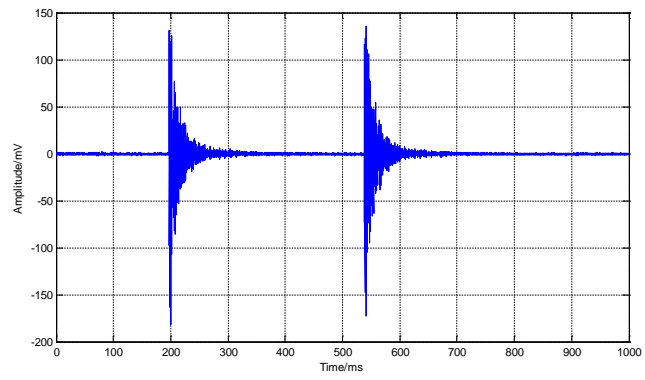

Fig. 6 Acoustic waveform of discharge between needle-plate gap

As can be seen from figures above, the waveform amplitude maintains at a very low level when there is no discharge in transformer oil. When the needle-plate gap is breakdown, a mutation appears in the waveform and soon declines. When the discharge acoustic signal is detected again, the waveform mutation will occur again.

PSD(Power Spectral Density) of the detected waveforms are calculated, and the results are shown in Figure 7 and 8. It is not difficult to find that the PSD achieves high value only in several specific frequency, while the PSD of other bands of frequency is generally at a lower level when there is no discharge. The PSD of acoustic signals is high in high frequency when discharge occurs, and it shows that discharge acoustic signals are mostly made of high frequency signals[6].

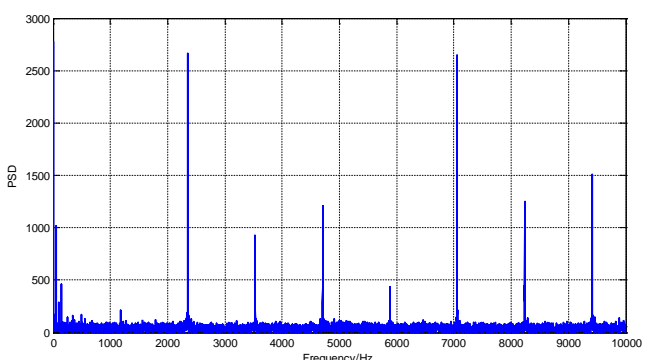

Fig. 7 PSD of acoustic signal when there's no discharge

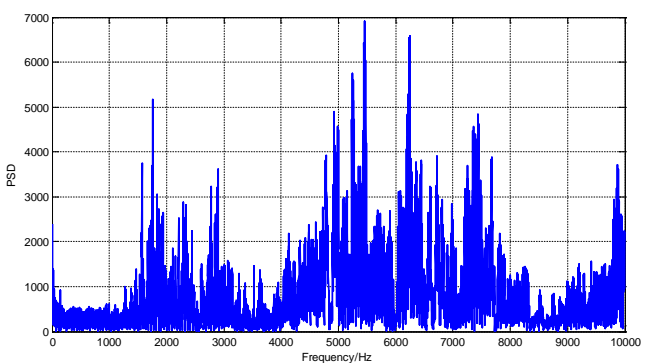

Fig. 8 PSD of acoustic signal of discharge between needle-plate gap

\section{Substation test}

The environment is bad around the transformer substation, especially the transformers in operation The noise is big and the electromagnetic interference is strong. In order to test the monitoring effect of the fault monitoring device in substation, a converter transformer of UHVDC converter station is chosen to be tested. The test is carried out when the gear of the tap changer is rising and falling[7].

In the field test, the sensor is installed close to the external wall of the converter transformer, and the PC terminal controls data acquisition. Figure 9 shows the acoustic signal of transformer before the tap changer operates. Figure 10 shows the acoustic signal when the tap changer is falling. Figure 11 shows the acoustic signal when the tap changer is rising. 


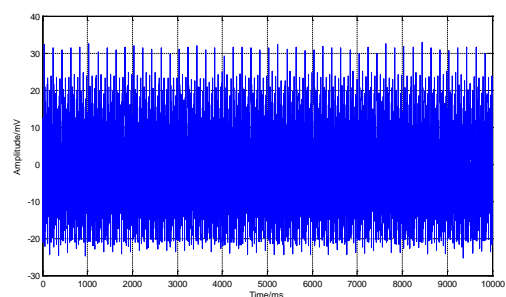

Fig. 9 Acoustic signal of transformer before tap changer operates

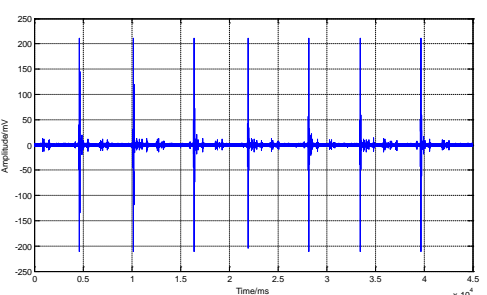

Fig. 10 Acoustic signal of transformer during the gear of tap changer is falling

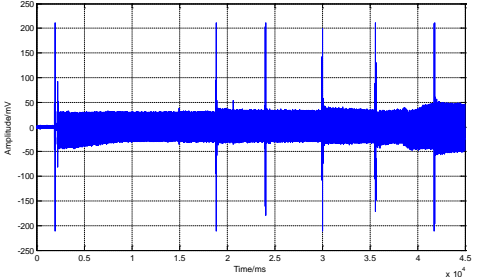

Fig. 11 Acoustic signal of transformer during the gear of tap changer is rising

As can be seen from the above 3 figures, the transformer fault monitoring device completes the acquisition of acoustic signals before and during the operation of the tap changer. In Figure 9, the signal is stable and the amplitude of the signal is low. In Figure 10, when the gear of tap changer is downshifting, a amplitude jump appears nearly every 5s, and the whole process lasts nearly 2 minutes. In Figure 11, acoustic signals shows different characteristics, that is the amplitude of the waveform of non-switch time in the process of gear-rising is significantly higher than that when the gear is falling.

\section{Conclusion}

Based on the analysis of the principle of acoustic signal generating from transformers, a transformer fault monitoring device based on acoustic characteristics is designed. The laboratory test and substation test show that the device can well monitor the abnormal acoustic signal of transformers. It achieves the purpose of on-line monitoring, and provides certain guide for transformer maintenance.

\section{References}

[1]A. Cichon, S. Borucki, T. Boczar, and M. Lorenc, "Results of the wavelet analysis of the acoustic emission signals generated by partial discharges in insulation oil of various temperature and flow speed," The European Physical Journal Special Topics, vol. 154, pp. 31-38, 2008.

[2]E. Grossmann and K. Feser, "Sensitive online PD-measurements of onsite oil/paper-insulated devices by means of optimized acoustic emission techniques (AET)," IEEE Transactions on Power Delivery, vol. 20, pp. 158-162, 2005.

[3]S. Markalous, S. Tenbohlen and K. Feser, "Detection and location of partial discharges in power transformers using acoustic and electromagnetic signals," EEE Transactions on Dielectrics and Electrical InsulationI, vol. 15, pp. 1576-1583, 2008-01-01 2008.

[4]L. E. Lundgaard, "Acoustic Partial Discharge Detection -Fundamental Considerations," IEEE Electrical Insulation Magazine, vol. 8, pp. 25-31, 1992.

[5]M. MacAlpine, Z. Zhiqiang and M. S. Demokan, "Development of a fibre-optic sensor for partial discharges in oil-filled power transformers," Electric Power Systems Research, vol. 63, pp. 27-36, 2002.

[6]E. D. Ubeyli and I. Guler, "Spectral analysis of internal carotid arterial Doppler signals using FFT, AR, MA, and ARMA methods," Computers in Biology and Medicine, vol. 34, pp. 293-306, 2004.

[7]T. B. Shanker, H. N. Nagamani and G. S. Punekar, "Acoustic emission signal analysis of On-Load Tap Changer (OLTC)," in 2013 IEEE 1st International Conference on Condition Assessment Techniques in Electrical Systems, 2013. 